\title{
miR-6835-3p regulates the function of pancreatic islet cells by modulating the expression of AdipoR1
}

\author{
HUIMIN WANG ${ }^{1}$, LEI JIANG ${ }^{2}$, ZHENFU LI $^{2}$, WEI WANG ${ }^{2}$ and CHUANJI HAO ${ }^{2}$ \\ Departments of ${ }^{1}$ Critical Care Medicine and ${ }^{2}$ Cardiac Surgery, The Affiliated Hospital \\ of Qingdao University, Qingdao, Shandong 266000, P.R. China
}

Received January 24, 2018; Accepted June 11, 2018

DOI: $10.3892 /$ ijmm.2018.3731

\begin{abstract}
Effective drugs and strategies for treating type 2 diabetes mellitus (2-DM) are urgently required. The aim of the present study was to elucidate the mechanism underlying microRNA (miR)-6835-3p regulation of adiponectin receptor 1 (AdipoR1) expression and the miR-6835-3p/AdipoR1 signaling pathway in pancreatic islet cells. In addition, the potential anti-diabetes effect of miR-6835-3p on insulin secretion was investigated. Luciferase activity analysis was performed to evaluate how miR-6835-3p targets the 3'-untranslated region of AdipoR1. The SU.86.86 and MIN-6 cell lines were co-cultured with or without miR-6835-3p inhibitors or mimics, and the insulin secretory functions of these cell lines were then determined. Luciferase reporter analysis revealed that AdipoR1 was a direct target of miR-6835-3p. In addition, miR-6835-3p overexpression suppressed the mRNA and protein expression levels of AdipoR1 in the SU.86.86 and MIN-6 cell lines. Furthermore, miR-6835-3p exerted negative effects on insulin secretion in SU.86.86 and MIN-6 cells, which were mediated by regulating AdipoR1 expression. AdipoR1 was a direct target of miR-6835-3p; therefore, inhibition of AdiopR1 expression may reduce insulin secretion and may be considered a key regulator of insulin secretion. The results of the present study suggested that targeting AdipoR1 with miR-6835-3p inhibitors may be a potential strategy for promoting glucose-stimulated insulin secretion, and thereby, may be an effective treatment for type 2-DM.
\end{abstract}

\section{Introduction}

Diabetes mellitus (DM) is caused by dysfunction of the endocrine system; this metabolic dysfunction is characterized by impaired metabolism of proteins, carbohydrates or fats,

Correspondence to: Dr Chuanji Hao, Department of Cardiac Surgery, The Affiliated Hospital of Qingdao University, 16 Jiangsu Road, Qingdao, Shandong 266000, P.R. China

E-mail: haochuanji@163.com

Key words: microRNA, glucose-stimulated insulin secretion, diabetes, adiponectin receptor 1 and hyperglycemia (1). Type 2-DM is a major public health concern, which is increasing in frequency worldwide. In addition, it is commonly associated with various complications and is a major chronic disease (2). It has been predicted that the prevalence of type 2-DM will continue to rise, with $>7.5 \%$ of the global population thought to be affected by 2030 (2). Therefore, more effective methods and therapeutic agents for treating type 2-DM and its associated complications are urgently required (3).

A growing body of evidence has indicated that microRNAs (miRNAs/miRs) serve important roles in the post-transcriptional regulation of gene expression (4). Transfection of cells with appropriate miRNAs is one way to induce the generation of pancreatic $\beta$-cells (5). The regulatory effects of miRNAs on cell proliferation, differentiation and fate indicate that miRNAs serve key roles in animal development (6-8). Embryonic stem cells could be differentiated into fully functional islet cells, which would be able to secrete insulin and thus, could be an alternative method to replace damaged pancreatic islet $\beta$-cells. In recent years, it has been indicated that the regeneration and differentiation of islet $\beta$-cells may be induced by regulating miRNA expression (9). Similarly, the development of islet $\beta$-cells has been revealed to be regulated by the miR-200 family and miR-30, which control the transition from epithelial to mesenchymal cells (9). The dynamic expression patterns of miR-375 and miR-7 in the stem cell differentiation process are similar to those of miR-146a and miR-34a expression in the developing pancreas of the human fetus (10). Furthermore, V-Maf avian musculoaponeurotic fibrosarcoma oncogene homolog $\mathrm{B}$ and Forkhead box (FoxO)-A2 are targeted by miR-342, which is also associated with the maturation and differentiation of islet $\beta$-cells (9). During the development and differentiation of $\beta$-cells, the key transcription factors associated with these processes are specifically targeted by miRNAs $(9,10)$. Therefore, miRNAs may be an efficient, alternative strategy for the regeneration of islet cells.

Type 2-DM has been consistently associated with hypoadiponectinemia, which is associated with adiponectin and its receptors. Therefore, the present study used the online software TargetScan (targetscan.org/) to predict the miRNAs that may regulate the expression of adiponectin receptor 1 (AdipoR1) in $\beta$-cells. It was subsequently identified that AdipoR 1 may be a potential target gene of miR-6835-3p. 
The present study used the MIN-6 pancreatic $\beta$-cell line (Mus musculus; transgenic for SV40 large T antigen), which was derived from insulinoma, and the SU.86.86 cell line (CRL-1837 ${ }^{\mathrm{TM}}$ ), which has pancreatic tissue origin (Homo sapiens) and was derived from metastatic ductal carcinoma of the liver. The aim of the present study was to evaluate the effects of miRNA on pancreatic cell function. The two pancreatic cell lines, SU.86.86 and MIN-6, were used to establish an appropriate model for in vitro functional experiments.

Previous research (7) performed by our group assessed the effects of miR-6835-3p on SU.86.86 and MIN-6 cell lines, and revealed that AdipoR1 mRNA contained one miR-6835-3p target sequence. The present results confirmed that AdipoR1 mRNA may be a target of miR-6835-3p in islet $\beta$-cells. In addition, AdipoR1 may affect the biological functions of the SU.86.86 and MIN-6 cell lines. Therefore, the present study aimed to investigate the in vitro effects of miR-6835-3p on the insulin secretory function of $\beta$-cells.

\section{Materials and methods}

Cell lines. The human pancreatic ductal carcinoma cell line SU.86.86 (CRL-1837) and the mouse pancreatic islet cell line MIN-6 were purchased from American Type Culture Collection (Manassas, VA, USA). Cells were cultured in Dulbecco's modified Eagle's medium (Gibco; Thermo Fisher Scientific, Inc., Waltham, MA, USA) supplemented with $15 \%$ heat-inactivated fetal bovine serum (Gibco; Thermo Fisher Scientific, Inc.), $25 \mathrm{mM}$ glucose and $5.5 \mathrm{mM}$ 2-mercaptoethanol. Cells were grown at $37^{\circ} \mathrm{C}$ in an incubator containing $5 \% \mathrm{CO}_{2}$. Lipofectamine ${ }^{\circledR} 3000$ (Invitrogen; Thermo Fisher Scientific, Inc.) was used for transfection experiments, according to the manufacturer's protocol (11).

Extraction of total RNA and miRNA. TRIzol ${ }^{\circledR}$ (Invitrogen; Thermo Fisher Scientific, Inc., Waltham, MA, USA) was used to extract total RNA from cells. The mirVana RNA isolation kit (Invitrogen; Thermo Fisher Scientific, Inc.) was used to remove RNA $<200 \mathrm{nt}$ from isolated total RNAs. Subsequently, cDNA was generated using the SMART-cDNA synthesis kit (Clontech Laboratories, Inc., Mountainview, CA, USA), which was conducted according to the manufacturer's protocol. In addition, the 3'-untranslated region (UTR) of AdipoR1 was cloned into the pmirGLO vector using the DynaExpress miRNA Cloning kit (BioDynamics Laboratory, Inc., Tokyo, Japan) (12).

Analysis of the reporter gene. The whole cDNA sequence that targets the 3'-UTR of AdipoR1 mRNA was predicted and obtained from the total RNA of the two cell lines. The reverse orientation of AdipoR1 3'-UTR was used as a control (13). Notably, GUGCUUUU was identified as the seed region of miR-6835-3p, which bound to the 3'-UTR region of AdipoR1 in position 38-44. After transfection with miR-6835-3p, the 3'-UTRs of AdipoR1, FoxO-1 and SIRT-1 were transfected into the two cell lines. Cells were transfected with miR-6835-3p mimics (Invitrogen; Thermo Fisher Scientific, Inc.) $\left(3 \mu \mathrm{g} / \mathrm{ml}\right.$ ) using Lipofectamine ${ }^{\circledR} 3000$ (Invitrogen; Thermo Fisher Scientific, Inc.) for $48 \mathrm{~h}$ at $37^{\circ} \mathrm{C}$, and were then transfected with the $3^{\prime}$-UTRs of AdipoR1 (4 $\left.\mu \mathrm{g} / \mathrm{ml}\right)$, FoxO-1 and
SIRT-1 based on the aforementioned method. The Dual-Glo ${ }^{\circledR}$ Luciferase Assay system (Promega Corporation, Madison, WI, USA) was used to determine luciferase reporter gene activity, according to the manufacturer's protocol. The Site-Mutation kit (Promega Corporation) was used to construct the mutant (mut) 3'-UTR of AdipoR1. Furthermore, FoxO-1 and SIRT-1 mRNA 3'UTRs were also obtained from the two cell lines, and luciferase reporter assays were conducted.

Transfection with miR-6835-3p inhibitors or mimics. For cells that were transduced with vectors and transfected with mimics/inhibitors, transduction was performed first. Lipofectamine $^{\circledR} 3000$ (Invitrogen; Thermo Fisher Scientific, Inc.) was used to perform transfection experiments, according to the manufacturer's protocol. The miR-6835-3p mimics (MC29537) and inhibitors (MH29537) were purchased from Thermo Fisher Scientific, Inc., respectively. In addition, the sequence of the negative control used was as follows: 5 '-ACG UGACACGUUCGGAGAAUU-3'. The effects of miR-6835-3p inhibitors or mimics on miR-6835-3p expression were evaluated using reverse transcription-quantitative polymerase chain reaction (RT-qPCR). The mimics/inhibitors were labeled with green fluorescent protein (GFP) to allow for observation, in order to verify whether cell transfection was successful (Fig. 1). The images were captured using a fluorescence microscope at x200 magnification (IX71; Olympus Corporation, Tokyo, Japan).

$R T$-qPCR assay. The mRNA expression levels of AdipoR1 were detected in the two cell lines using RT-qPCR. Briefly, RT-qPCR was performed with SYBR Green (Sigma-Aldrich; Merck KGaA, Darmstadt, Germany) and the Light-Cycler Roche 480 system (Roche Diagnostics, Basel, Switzerland), The thermocycling conditions for qPCR were as follows: Pre-denaturation at $95^{\circ} \mathrm{C}$ for $30 \mathrm{sec}$, followed by 40 cycles of denaturation at $95^{\circ} \mathrm{C}$ for $5 \mathrm{sec}$ and extension at $60^{\circ} \mathrm{C}$ for $30 \mathrm{sec}$. The $2^{-\Delta \Delta C a}$ method was used to calculate relative expression and quantify $(12,14)$. The primer sequences used were as follows: AdipoR1, forward 5'-GCAGGCACATTACACGGT-3', reverse 5'-TCCAGTTTTTTTTTTTTTTTAGAGGTC-3'; GAPDH forward 5'-CTCATGACCACAGTCCATGCC-3', reverse 5'-GGCATGGACTGTGGTCATGAG-3'; miR-6835-3p, forward 5'-GACCCTCTGTCTTTTCACGAAAA-3', reverse 5'-TTTTCGTGAAAAGACAGAGGGTC-3'; and U6, forward 5'-CTCGCTTCGGCAGCACA-3' and reverse 5'-TGTGCT GCCGAAGCGAG-3'.

Western blot analysis. Cells were collected and total proteins were extracted in $40 \mathrm{mM}$ Tris- $\mathrm{HCl}(\mathrm{pH}$ 7.4) containing $150 \mathrm{mM} \mathrm{NaCl}$ and 1\% (v/v) Triton X-100 (Sigma-Aldrich; Merck KGaA), supplemented with protease inhibitors (Sigma-Aldrich; Merck KGaA). Protein concentration was determined using the bicinchoninic acid protein assay (Pierce; Thermo Fisher Scientific, Inc.). Total cell proteins were extracted and separated by $12 \%$ SDS-PAGE; $50 \mu$ g protein was loaded per lane. Proteins were then transferred to a $0.45-\mu \mathrm{m}$ polyvinylidene fluoride membrane (Roche Diagnostics), which was blocked with $1 \mathrm{X}$ Tris-buffered saline-Tween-20 $(0.05 \%)$ containing $5 \%$ nonfat dry milk and agitated for $1 \mathrm{~h}$ at room temperature. The membranes were then incubated with primary antibodies against AdipoR1 (1:2,000; sc-518030), 


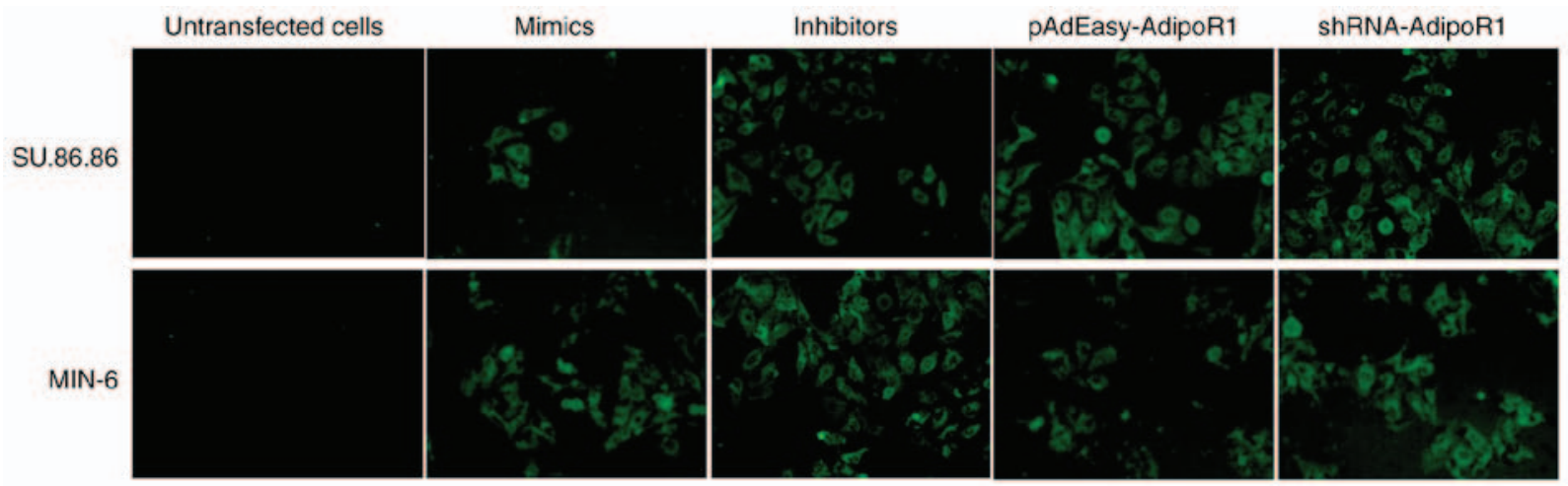

Figure 1. Confirmation that cell transfection with mimics/inhibitors of microRNA-6835-3p was successful. The mimics/inhibitors were labeled with green fluorescent protein to allow for observation and images were captured with a fluorescence microscope at x200 magnification (IX71; Olympus Corporation, Tokyo, Japan), in order to verify whether cell transfection was successful. shRNA, short hairpin RNA.

Sirtuin 1 (SIRT-1) (1:2,000; sc-135791), FoxO-1 (1:2,000; sc-9808; Santa Cruz Biotechnology, Inc., Dallas, TX, USA) and $\beta$-actin (1:3,000 dilution; 4970; Cell Signaling Technology, Inc., Danvers, MA, USA) at $4^{\circ} \mathrm{C}$ overnight. The membranes were then incubated with secondary antibodies goat anti-rabbit immunoglobulin $\mathrm{G}$ (IgG)-horseradish peroxidase (HRP) (sc-2004; 1:5,000 dilution; Santa Cruz Biotechnology, Inc.) or goat anti-mouse IgG-HRP, (sc-2005; 1:5,000 dilution; Santa Cruz Biotechnology, Inc.) for $1 \mathrm{~h}$ at room temperature. Protein bands were visualized using enhanced chemiluminescence (Amersham; GE Healthcare, Chicago, IL, USA).

Transduction of AdipoRl and its short hairpin (sh)RNA. For transduction of AdipoR1, PCR amplification was performed for AdipoR1, and its open-reading frame was cloned into the pAdEasy vector (Stratagene; Agilent Technologies, Inc., Santa Clara, CA, USA). To generate and amplify the virus, 293A cells (Thermo Fisher Scientific, Inc.) were employed; cells $(90 \%$ confluence) were transfected with vectors $(4 \mu \mathrm{g} / \mathrm{ml})$ using Lipofectamine ${ }^{\circledR} 3000$ (Invitrogen; Thermo Fisher Scientific, Inc.) for $48 \mathrm{~h}$ at $37^{\circ} \mathrm{C}$. Purification was subsequently performed using the double cesium chloride gradient centrifugation method (15). The 293A cells were harvested, and adenovirus stocks were prepared by repeated freezing and thawing. The infectious particles were measured in culture by a plaque-forming unit (PFU) assay using a Cell Biolabs QuickTiter ${ }^{\mathrm{TM}}$ Adenovirus Titer Immunoassay kit (VPK-109; Cell Biolabs, Inc., San Diego, CA, USA), which scores the number of viral plaques as a function of dilution. The adenovirus titer reached $\sim 3.5 \times 10^{9} \mathrm{pfu} / \mathrm{ml}$. Subsequently, the viral supernatant $(1 \mathrm{ml})$ was used to infect target cells ( $2 \times 10^{6}$ cells) for 10 days. AdipoR1 shRNA (5'-GGACAACGA CUAUCUGCUACATT-3') and the negative control shRNA (shRNA-control; 5'-CCUACGCCACCAAUUUCGU-3') were obtained from Shanghai GenePharma Co., Ltd. (Shanghai, China), and were prepared via ligation of the corresponding pairs of oligonucleotides to the pLKO.1 vector (Sigma-Aldrich; Merck KGaA). The pLKO.1 vectors containing AdipoR1 shRNA or shRNA-control were introduced into target cells using Lipofectamine ${ }^{\circledR} 3000$ (Invitrogen; Thermo Fisher Scientific, Inc.) for $48 \mathrm{~h}$ at $37^{\circ} \mathrm{C}$. The AdipoR1 vectors were labeled with GFP for observation, in order to verify successful transduction/transfection into cells (Fig. 1).
Insulin secretion assay. The insulin secretion assay was conducted as previously described $(16,17)$. Briefly, following a $2 \mathrm{~h}$ preincubation period in the modification medium [Krebs-Ringer (Sigma-Aldrich; Merck KGaA) containing $3 \mathrm{mmol} / \mathrm{l}$ glucose], cells were treated with $20 \mathrm{mM}$ glucose for $1 \mathrm{~h}$. Subsequently, the insulin levels in the cultured cell supernatant fractions were determined using the radioimmunoassay method using a specific insulin radioimmunoassay kit (cat. no. RI-13K; Linco Research, Inc., St. Charles, MO, USA).

Statistical analysis. A two-tailed paired Student's t-test was used to analyze differences between two groups, and one-way analysis of variance (ANOVA) was performed for multiple comparisons between $>2$ groups; when the variances were equal, the least signidicant difference post hoc test was used, whereas when the variances were not uniform, Dunnett's post hoc test was used. All data are presented as the means \pm standard deviation. SPSS software (version 18.0; SPSS, Inc., Chicago, IL, USA) was used to conduct statistical analyses. $\mathrm{P}<0.05$ was considered to indicate a statistically significant difference.

\section{Results}

miR-6835-3p inhibits AdipoR1 expression in SU.86.86 and MIN-6 cells. The present study used TargetScan software (targetscan.org/) to predict the miRNA that may regulate the expression of AdipoR1 in SU.86.86 cells. Subsequently, AdipoR1 was identified as a potential target gene of miR-6835-3p (Fig. 2). Reporter gene assays based on luciferase activity were performed to confirm that the AdipoR1 3'-UTR was able to bind directly to miR-6835-3p. The results indicated that miR-6835-3p markedly downregulated 3'-UTR AdipoR1 luciferase activity in SU.86.86 and MIN-6 cells; however, the luciferase activity of the mut-AdipoR 1 group was not influenced by miR-6835-3p (Fig. 3A and B). In addition, the results confirmed that miR-6835-3p did not directly bind to FoxO-1 or SIRT-1 3'-UTRs (Fig. 3C and D). These findings indicated that AdipoR1 mRNA was the direct target of miR-6835-3p.

In order to determine whether miR-6835-3p affected the mRNA and protein expression levels of FoxO-1, SIRT-1 and AdipoR1 in SU.86.86 and MIN-6 cells, RT-qPCR and western 


\begin{tabular}{|c|c|c|c|c|c|c|c|}
\hline & $\begin{array}{l}\text { Predicted consequential pairing of target region (top) } \\
\text { and miRNA (bottom) }\end{array}$ & $\begin{array}{l}\text { Site } \\
\text { type }\end{array}$ & $\begin{array}{l}\text { Context++ } \\
\text { score }\end{array}$ & $\begin{array}{l}\text { Context++ score } \\
\text { percentile }\end{array}$ & $\begin{array}{l}\text { Weighted } \\
\text { context++ score }\end{array}$ & $\begin{array}{c}\text { Conserved branch } \\
\text { length }\end{array}$ & PCT \\
\hline $\begin{array}{l}\text { Position } 38-44 \text { of ADIPOR1 } 3^{\prime} \text { UTR } \\
\text { hsa-miR-6835-3p }\end{array}$ & $\begin{array}{l}5^{\prime} \quad \text {...GGAGGAACUUCCCAAGUGCUUUU... } \\
\text { 3' }^{\prime} \quad \text { GACCCUCUGUCUUUUCACGAAAA }\end{array}$ & $\begin{array}{c}\text { 7mer- } \\
\mathrm{m} 8\end{array}$ & -0.29 & 99 & -0.29 & 3.018 & N/A \\
\hline
\end{tabular}

Figure 2. Predicted results of AdipoR1 regulation by miR-6835-3p using TargetScan. The online software TargetScan was used to predict the miRNA target of AdipoR1. AdipoR1 was subsequently identified as a potential target gene of miR-6835-3p. AdipoR1, adiponectin receptor 1; miRNA/miR, microRNA.
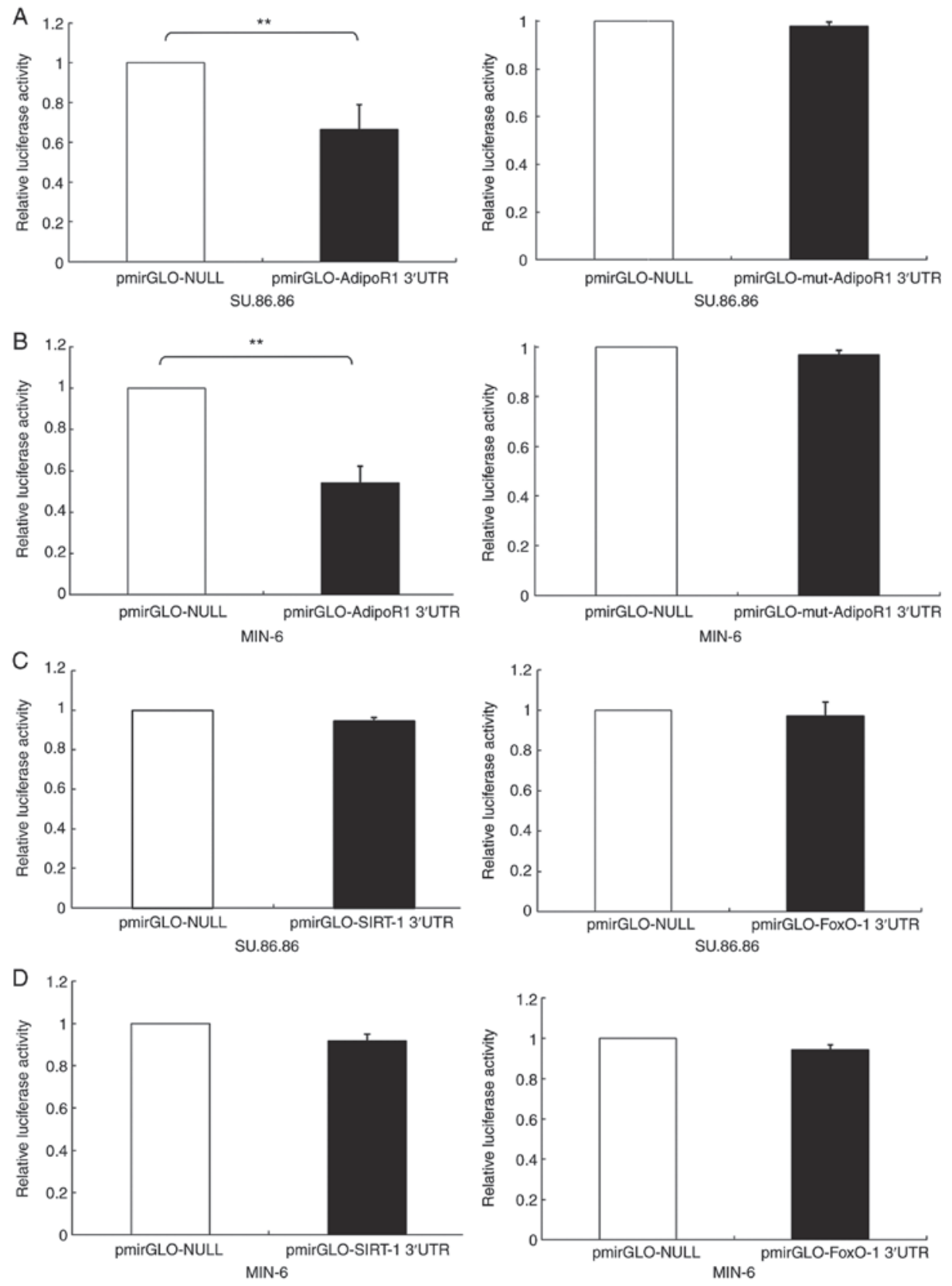

Figure 3. Relative luciferase activities of AdipoR1 were suppressed by miR-6835-3p in SU.86.86 and MIN-6 cells. The results revealed that the relative luciferase activities of AdipoR1 3'-UTR were markedly downregulated in (A) SU.86.86 and (B) MIN-6 cells transfected with miR-6835-3p; no change was observed in the mut-AdipoR1 group. Conversely, miR-6835-3p did not directly bind to the SIRT-1 or FoxO-1 3'-UTRs in (C) SU.86.86 and (D) MIN-6 cells. Data are presented as the means \pm standard deviation from three independent experiments. ${ }^{* *} \mathrm{P}<0.01$. AdipoR1, adiponectin receptor 1 ; FoxO-1, Forkhead box O1; miR, microRNA; mut, mutant; UTR, untranslated region; SIRT-1, Sirtuin 1.

blotting were performed (Fig. 4). The results demonstrated that miR-6835-3p suppressed the mRNA and protein expression levels of AdipoR1 in SU.86.86 and MIN-6 cells (Fig. 4A and B). Conversely, miR-6835-3p did not affect the mRNA expression levels of FoxO-1 and SIRT-1 (Fig. 4B); however, the protein expression levels of FoxO-1 and SIRT-1 were decreased. Furthermore, miR-6835-3p inhibitors facilitated the expression of AdipoR1 protein in the SU.86.86 and MIN-6 cell lines (Fig. 4B). Moreover, miR-6835-3p inhibitors enhanced the expression of AdipoR1 protein in the SU.86.86 and MIN-6 

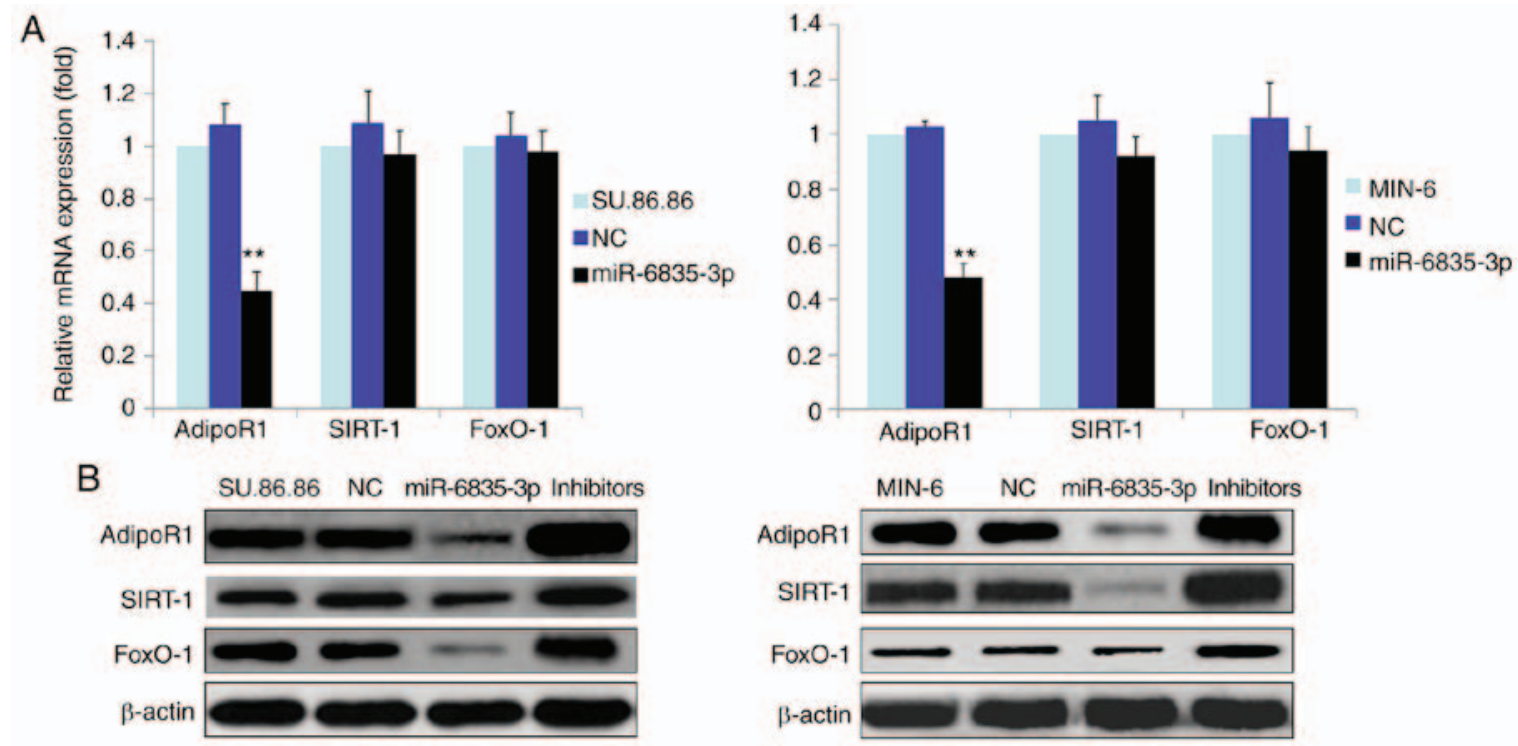

Figure 4. Expression of AdipoR1 is suppressed by miR-6835-3p in SU.86.86 and MIN-6 cell lines. The results indicated that miR-6835-3p inhibited the (A) mRNA and (B) protein expression levels of AdipoR1 in SU.86.86 and MIN-6 cells. miR-6835-3p did not affect the mRNA expression levels of SIRT-1 and FoxO-1; however, the protein expression levels of SIRT-1 and FoxO-1 were markedly decreased. Furthermore, miR-6835-3p inhibitors promoted the protein expression levels of AdipoR1. Data are presented as the means \pm standard deviation from three independent experiments. ${ }^{* *} \mathrm{P}<0.01$ compared with $\mathrm{SU} .86 .86$ and MIN-6 cells. AdipoR1, adiponectin receptor 1; miR, microRNA; NC, negative control; SIRT-1, Sirtuin 1; FoxO-1, Forkhead box O1.

cell lines (Fig. 4B). Taken together, these findings indicated that miR-6835-3p may directly modulate AdipoR1 expression by binding to the AdipoR1 3'-UTR, and the protein expression levels of FoxO-1 and SIRT-1 may be altered in response to miR mimics.

miRNA-6835-3p regulates glucose-stimulated insulin secretion (GSIS) via the AdipoR1 signaling pathway. SU.86.86 and MIN-6 were cultured with glucose $(20 \mathrm{mM})$, after which RT-qPCR was performed to detect the expression of miR-6835-3p. The results revealed that the expression levels of miR-6835-3p were lower in the high glucose-stimulated (20 mM) SU.86.86 and MIN-6 cell groups compared with in the control group (Fig. 5A and B), thus indicating that the expression of miR-6835-3p changed in response to glucose stimulation. In addition, the effects of miR-6835-3p mimics or inhibitors on GSIS were investigated. The results indicated that miR-6835-3p mimics suppressed GSIS; however, the inhibitors promoted GSIS in the SU.86.86 and MIN-6 cell lines (Fig. 5C and D).

Subsequently, the effects of AdipoR1 on GSIS in the SU.86.86 and MIN-6 cell line were determined (Figs. 6 and 7). It was demonstrated that AdipoR1 overexpression regulated GSIS in SU.86.86 and MIN-6 cells (Figs. 6A and 7A). To confirm the effects of AdipoR1 on GSIS, the present study employed an AdipoR1 shRNA, and its effects on AdipoR1 expression were confirmed in SU.86.86 and MIN-6 cells (Figs. 6B and 7B). The results demonstrated that knockdown of AdipoR1 inhibited insulin secretion stimulated by high levels of glucose in SU.86.86 and MIN-6 cells (Figs. 6C and 7C). Furthermore, the effects of glucose on the expression of AdipoR1 were assessed. The relative mRNA expression levels of AdipoR1 were increased in the high glucose-stimulated (20 mM) SU.86.86 and MIN-6 cell groups compared with in the control groups (Figs. 6D and 7D).
To confirm the effects of AdipoR1 on glucose-stimulated insulin secretion, the present study transduced SU.86.86 and MIN-6 cells with a shRNA for AdipoR1 and tested its efficiency. AdipoR1 shRNA downregulated the protein expression levels of AdipoR1, as well as those of SIRT1 and FoxO-1 in its downstream signaling pathway, in the MIN-6 and SU.86.86 cell lines (Fig. 8). These results confirmed that the AdipoR1 shRNA regulates the protein expression levels of AdipoR1 and its associated pathway proteins in the MIN-6 and SU.86.86 cell lines.

Since miR-6835-3p and AdipoR1 regulate GSIS, and AdipoR1 mRNA is a direct target of miR-6835-3p, the present study further investigated how the effects of miR-6835-3p on AdipoR1 expression were associated with GSIS (Figs. 9 and 10). As shown in Figs. 9A and 10A, overexpression of AdipoR1 abolished the effects of miR-6835-3p mimics on GSIS in the SU.86.86 and MIN-6 cell lines, respectively. Conversely, treatment with miR-6835-3p mimics and AdipoR1 shRNA synergistically inhibited GSIS (Figs. 9B and 10B). The effects of the miR-6835-3p/AdipoR1 signaling pathway on GSIS were further evaluated using miR-6835-3p inhibitors. The results demonstrated that AdipoR1 overexpression significantly enhanced the effects of miR-6835-3p inhibitors on GSIS (Figs. 9C and 10C). In addition, GSIS was reduced in the SU.86.86 and MIN-6 cell lines treated with miR-6835-3p inhibitors and AdipoR1 shRNA compared with in cells transfected with miR-6835-3p inhibitors alone (Figs. 9D and 10D). Taken together, these results confirmed that AdipoR1 was a direct target of miR-6835-3p, and targeting AdipoR1 with miR-6835-3p inhibitors could promote GSIS.

\section{Discussion}

Type 2-DM is associated with blindness, renal failure and cardiovascular disease $(16,18)$. The increasing incidence of 

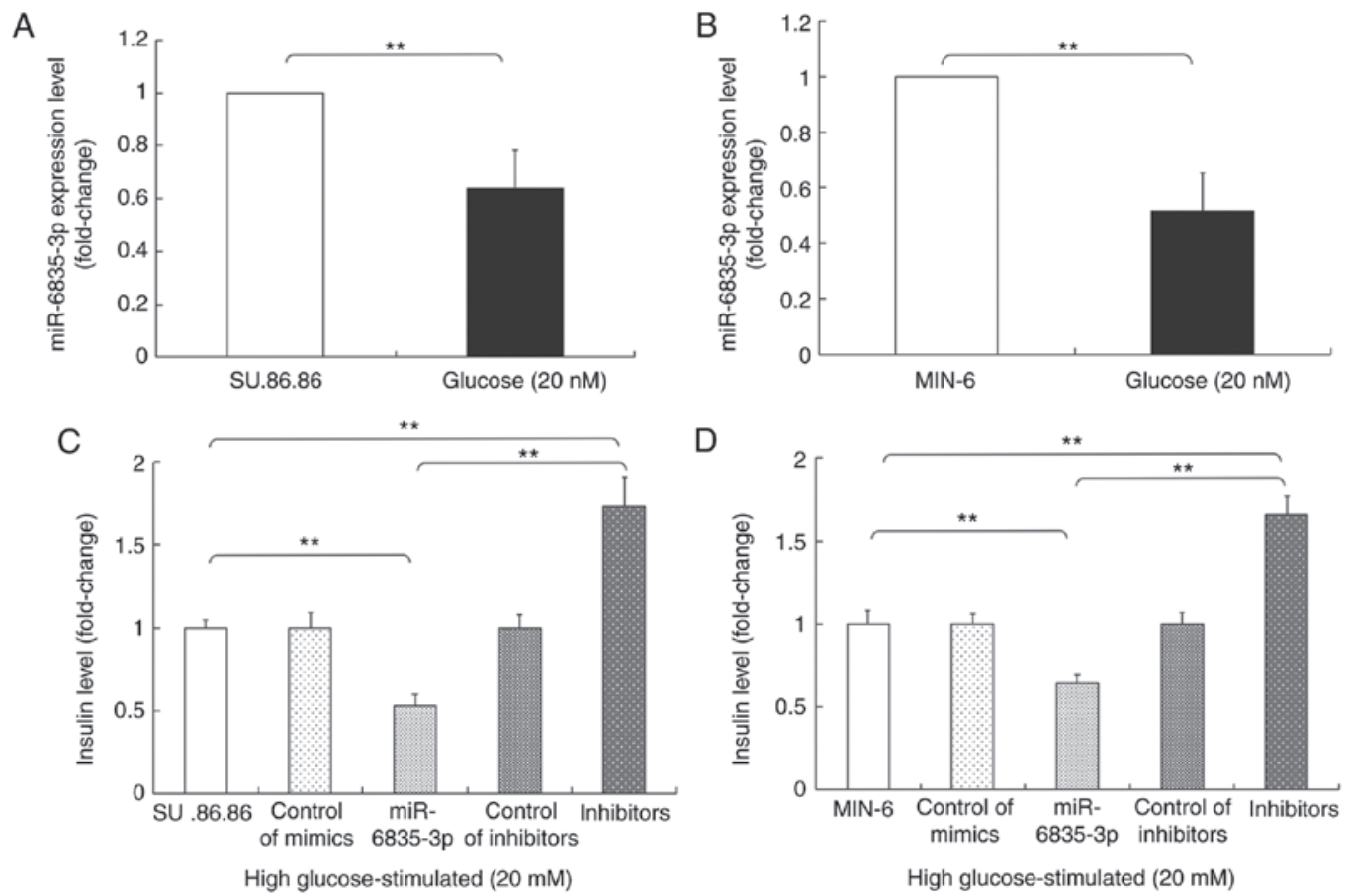

Figure 5. miRNA-6835-3p regulates GSIS. SU.86.86 and MIN-6 cells were incubated with $20 \mathrm{mM}$ glucose for $24 \mathrm{~h}$, and reverse transcription-quantitative polymerase chain reaction was performed. (A and B) Expression levels of miR-6835-3p in high glucose-stimulated (20 mM) SU.86.86 and MIN-6 cells were lower than in the control group. (C and D) Further investigation of the role of miR-6835-3p in GSIS was then performed. The results indicated that miR-6835-3p mimics suppressed GSIS, whereas miR-6835-3p inhibitors promoted GSIS in SU.86.86 and MIN-6 cells. Data are presented as the means \pm standard deviation from three independent experiments. ${ }^{* *} \mathrm{P}<0.01$. GSIS, glucose-stimulated insulin secretion; miRNA/miR, microRNA.
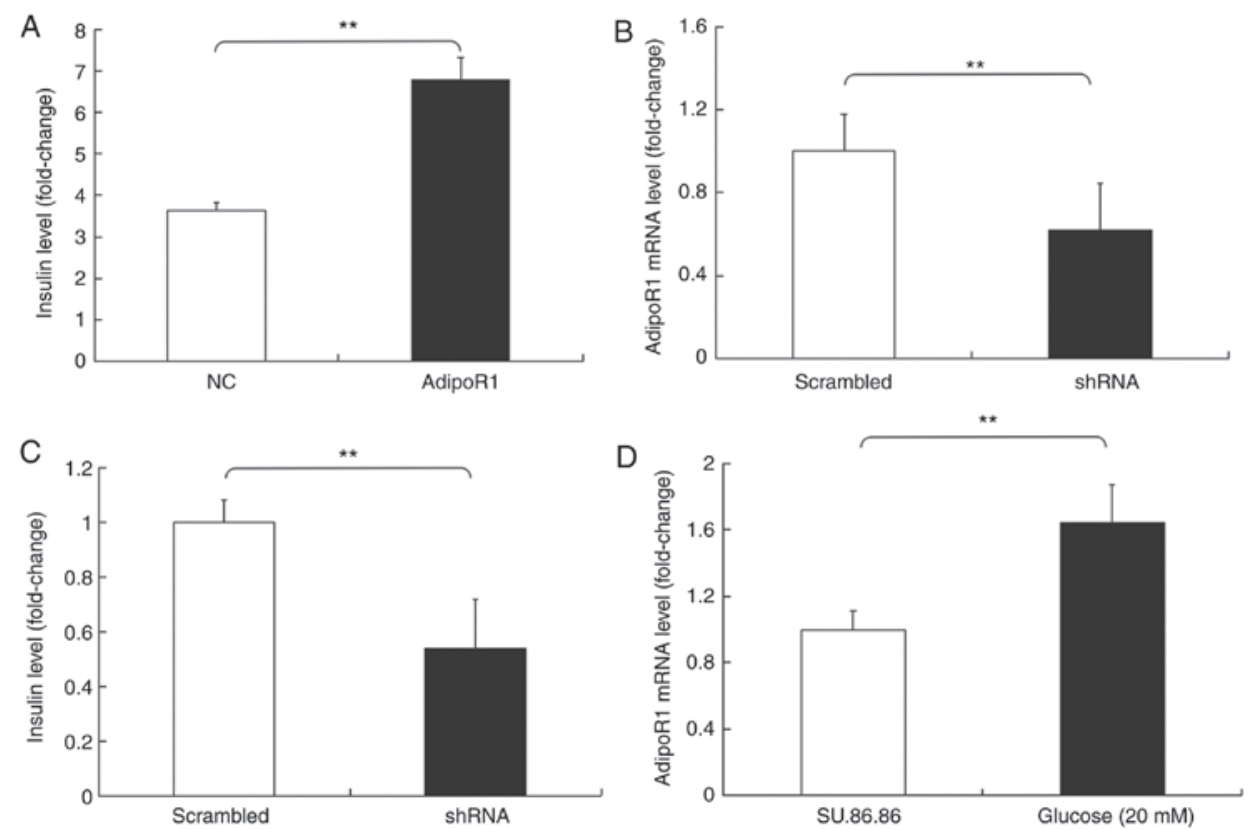

Figure 6. AdipoR1 regulates GSIS in the SU.86.86 cell line. (A) AdipoR1 overexpression regulated GSIS in SU.86.86 cells. (B) To confirm the effects of AdipoR1 on GSIS, the present study employed an AdipoR1 shRNA and evaluated its efficiency in SU.86.86 cells. (C) AdipoR1 knockdown inhibited insulin secretion stimulated by high levels of glucose in SU.86.86 cells. (D) Effects of glucose on AdipoR1 expression were also assessed. The relative mRNA expression levels of AdipoR1 were greater in the high glucose-stimulated SU.86.86 cell group compared with in the control group. Data are presented as the means \pm standard deviation from three independent experiments. ${ }^{* *} \mathrm{P}<0.01$. AdipoR1, adiponectin receptor 1; GSIS, glucose-stimulated insulin secretion; NC, negative control; shRNA, short hairpin RNA.

type 2-DM is a worldwide phenomenon, which coincides with the lifestyle changes of the last few decades $(19,20)$. Insulin is a small peptide hormone that is crucial for glucose homeostasis in mammals and is generated from pancreatic islet $\beta$-cells (21-23). In healthy mammals, when blood glucose concentration is elevated, insulin is released to promote the absorption of glucose by fat tissues, skeletal muscles or the liver $(24,25)$. 

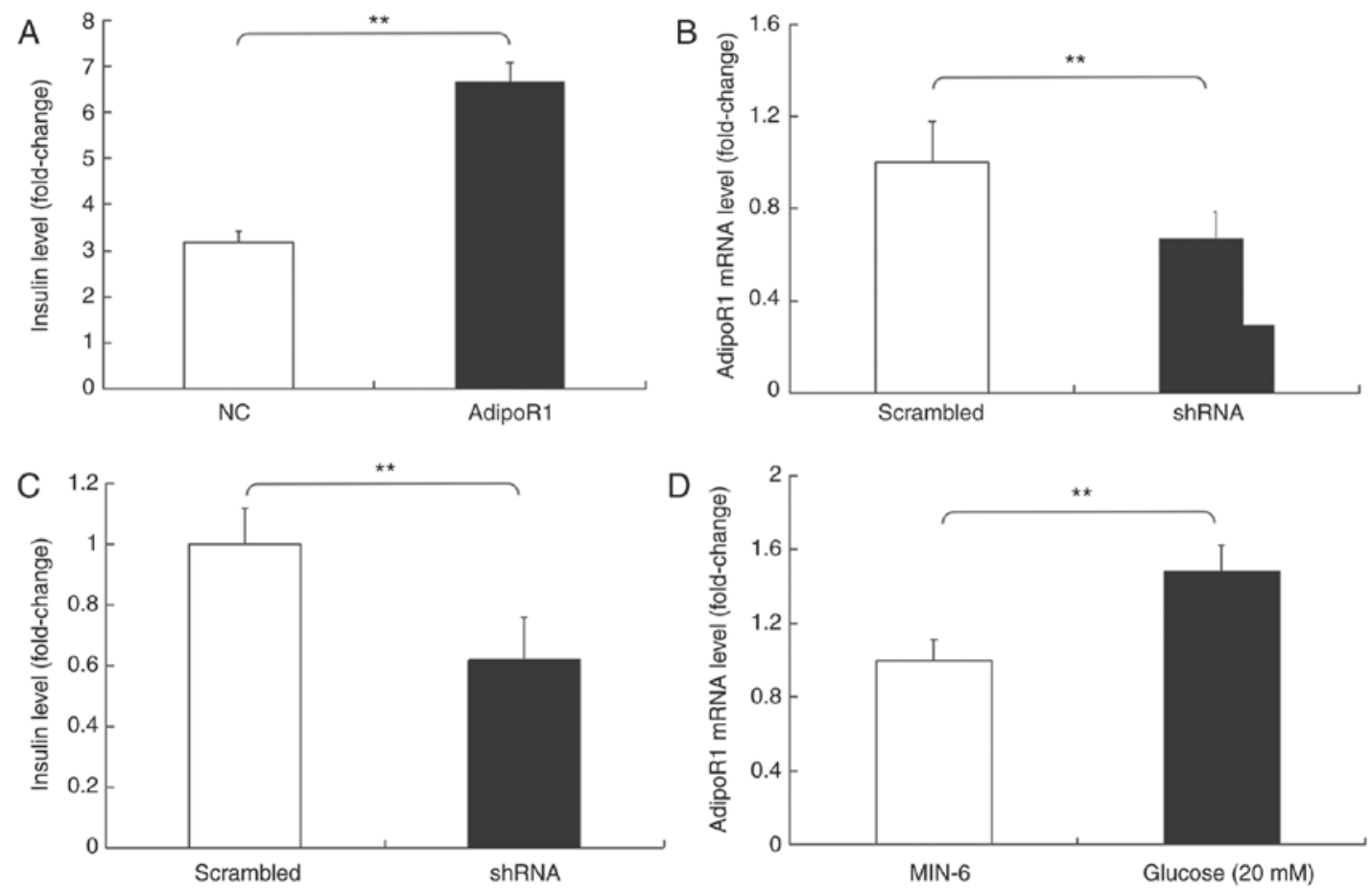

Figure 7. AdipoR1 regulates GSIS in the MIN-6 cell line. (A) AdipoR1 overexpression regulated GSIS in MIN-6 cells. (B) To confirm the effects of AdipoR1 on GSIS, the present study employed an AdipoR1 shRNA and evaluated its efficiency in MIN-6 cells. (C) AdipoR1 knockdown inhibited insulin secretion stimulated by high levels of glucose in MIN-6 cells. (D) Effects of glucose on AdipoR1 expression were also assessed. The relative mRNA expression levels of AdipoR1 were greater in the high glucose-stimulated MIN-6 cell group compared with in the control group. Data are presented as the means \pm standard deviation from three independent experiments. ${ }^{* *} \mathrm{P}<0.01$. AdipoR1, adiponectin receptor 1; GSIS, glucose-stimulated insulin secretion; $\mathrm{NC}$, negative control; shRNA, short hairpin RNA.
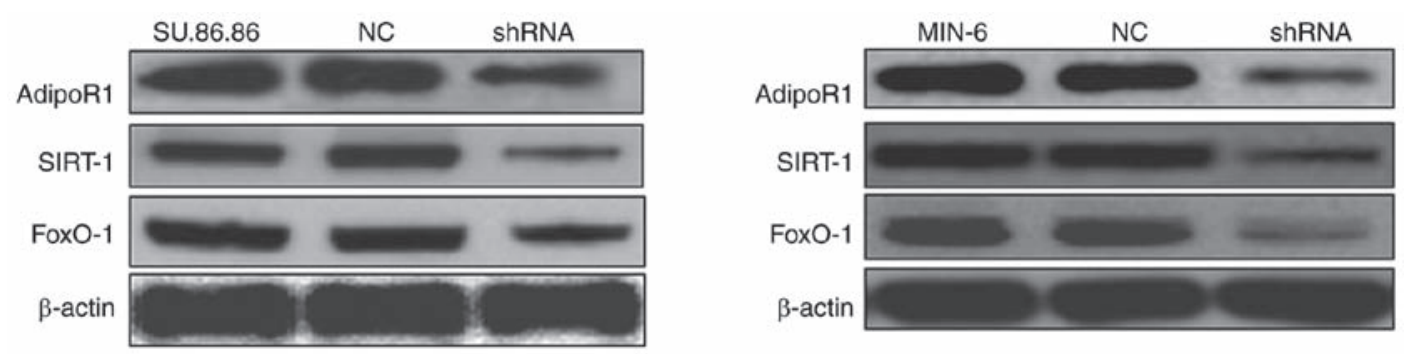

Figure 8. AdipoR1 shRNA regulates the protein expression levels of AdipoR1 and its associated pathway proteins in the MIN-6 and SU.86.86 cell lines. To confirm the effects of AdipoR1 on glucose-stimulated insulin secretion, the present study transduced SU.86.86 and MIN-6 cells with a shRNA for AdipoR1 and tested its efficiency. AdipoR1 shRNA downregulated the protein expression levels of AdipoR1, as well as those of SIRT1 and FoxO-1 in its downstream signaling pathway, in the MIN-6 and SU.86.86 cell lines. AdipoR1, adiponectin receptor 1; FoxO-1, Forkhead box O1; NC, negative control; shRNA, short hairpin RNA; SIRT-1, Sirtuin 1.

miRNAs are a class of 19-22 nucleotide-long endogenous RNAs, which can affect gene expression by binding to the 3'-UTRs of target mRNAs $(26,27)$. It has been indicated that miRNAs may be involved in pancreatic development and insulin secretion. In particular, miR-375 specifically inhibits GSIS and serves a vital role in insulin secretion (25); miR-9 regulates GSIS of islet $\beta$-cells by targeting SIRT- 1 in the pancreas (22); miR-124a is highly expressed in the pancreatic islets of patients with type 2-DM and inhibits insulin secretion (28); and miR-187 regulates the expression of homeodomain interacting protein kinase 3 , and is associated with reduced GSIS (21). In addition, miR-34c reduces GSIS by targeting vesicle-associated membrane protein 2, which induces vital effects of insulin secretion on $\beta$-cell exocytosis (29). Although numerous studies have reported that miRNAs target genes associated with GSIS signaling and have revealed their physiological implications, the underlying mechanisms associated with the modulation of GSIS have not been fully elucidated. In the present study, the roles of miR-6835-3p in GSIS and regulation of the AdipoR1/SIRT-1/FoxO-1 signaling pathway were investigated.

In insulin-resistant obesity, adiponectin is downregulated, as are adipokine factors secreted by adipose tissue. Type 2-DM, metabolic syndrome, atherosclerosis and obesity are consistently associated with hypoadiponectinemia (30). Adiponectin has been reported to induce anti-atherosclerotic and anti-diabetic effects by attenuating inflammation and oxidative stress $(31,32)$. The biological effects induced by adiponectin have been revealed to be associated with its 

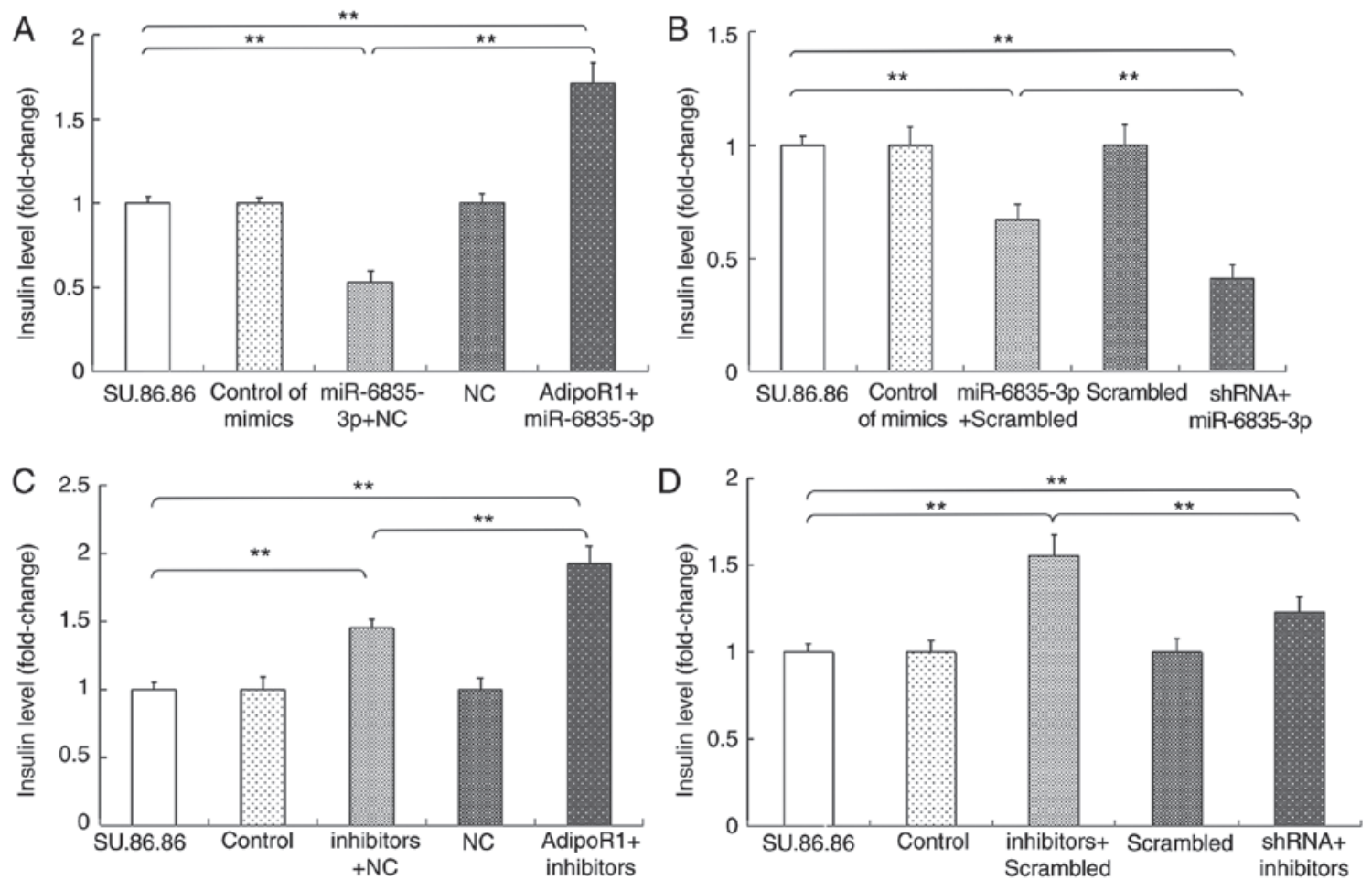

Figure 9. miR-6835-3p and AdipoR1 regulate GSIS in the SU.86.86 cell line. (A) Overexpression of AdipoR1 abolished the effects of miR-6835-3p mimics on GSIS. (B) Conversely, miR-6835-3p mimics and AdipoR1 shRNA synergistically inhibited GSIS. The effects of miR-6835-3p/AdipoR1 on GSIS were further evaluated using miR-6835-3p inhibitors. (C) AdipoR1 overexpression significantly enhanced the effects of miR-6835-3p inhibitors on GSIS. (D) Conversely, GSIS levels were reduced by miR-6835-3p inhibitors in the SU.86.86 cell line following AdipoR1 knockdown with the shRNA-AdipoR1. Data are presented as the means \pm standard deviation from three independent experiments. ${ }^{* *} \mathrm{P}<0.01$. AdipoR1, adiponectin receptor 1; GSIS, glucose-stimulated insulin secretion; miR, microRNA; NC, negative control; shRNA, short hairpin RNA.
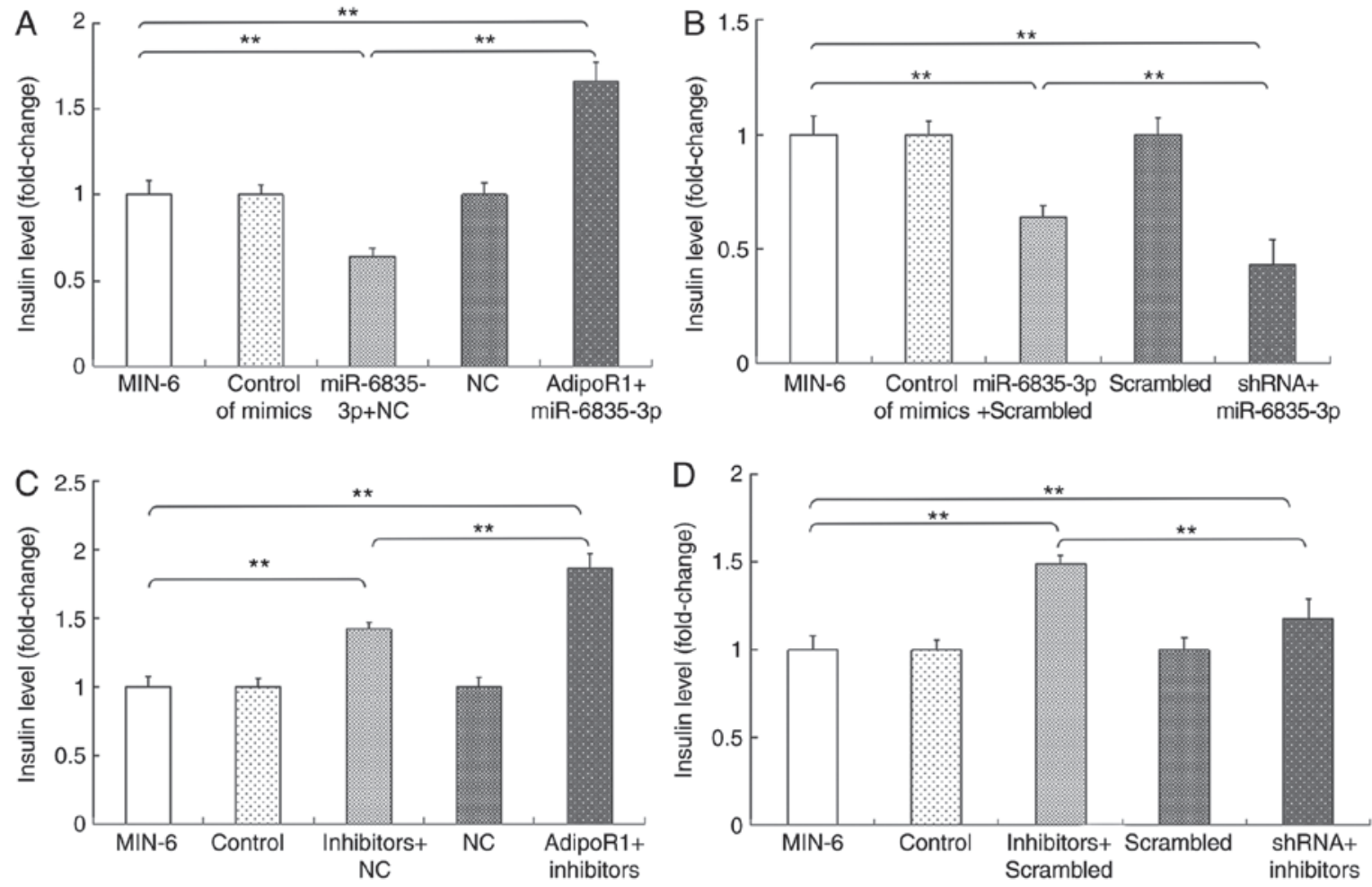

Figure 10. miR-6835-3p and AdipoR1 regulate GSIS in the MIN-6 cell line. (A) Overexpression of AdipoR1 abolished the effects of miR-6835-3p mimics on GSIS. (B) Conversely, miR-6835-3p mimics and AdipoR1 shRNA synergistically inhibited GSIS. The effects of miR-6835-3p/AdipoR1 on GSIS were further evaluated using miR-6835-3p inhibitors. (C) AdipoR1 overexpression significantly enhanced the effects of miR-6835-3p inhibitors on GSIS. (D) Conversely, GSIS levels were reduced by miR-6835-3p inhibitors in the MIN-6 cell line following AdipoR1 knockdown with shRNA-AdipoR1. Data are presented as the means \pm standard deviation from three independent experiments. ${ }^{* *} \mathrm{P}<0.01$. AdipoR1, adiponectin receptor 1; GSIS, glucose-stimulated insulin secretion; miR, microRNA; NC, negative control; shRNA, short hairpin RNA. 
receptors, AdipoR1 and AdipoR2. In addition, AdipoR1 is able to modulate SIRT1-FoxO1 signaling; a previous study revealed that resveratrol increases the phosphorylation of 5' AMP-activated protein kinase and SIRT-1, and decreases phosphorylation of downstream effectors FoxO-1 and FoxO-3a via increasing AdipoR1 and AdipoR2 in the renal cortex (33). In addition, capsaicin-activated FoxO-1 induces FoxO-1 acetylation, which is associated with CREB binding protein and SIRT-1 (34). SIRT-1 activation may induce FoxO1 activation, and it affects insulin function through modulation of the SIRT1-FoxO1 signaling axis (34). These findings indicated that AdipoR1/SIRT-1/FoxO-1 signaling axis may have an important role in insulin function. However, miRNA-mediated modulation of AdipoR 1 activity in the anti-diabetes process remains unclear.

In the present study, the miRNAs that may regulate AdipoR1 expression in the SU.86.86 and MIN-6 cell lines were predicted using TargetScan; AdipoR1 was identified as a potential target gene of $\mathrm{miR}-6835-3 \mathrm{p}$. The present results also indicated that miR-6835-3p markedly downregulated the luciferase activities of the AdipoR1 3'-UTR in SU.86.86 and MIN-6 cells; however, the luciferase activities of the mut-AdipoR1 group were not affected by miR-6835-3p. In addition, the results of a luciferase reporter assay confirmed that miR-6835-3p did not directly bind to the FoxO-1 or SIRT-1 3'-UTRs. Therefore, the present study indicated that AdipoR1 mRNA was the direct target of miR-6835-3p.

Further investigations demonstrated that miR-6835-3p suppressed the mRNA and protein expression levels of AdipoR1 in the SU.86.86 and MIN-6 cell lines; however, miR-6835-3p did not affect the mRNA expression levels of FoxO-1 and SIRT-1. Conversely, the protein expression levels of FoxO-1 and SIRT-1 were affected by miR-6835-3p. In addition, transfection with miR-6835-3p inhibitors facilitated the protein expression levels of AdipoR1 in SU.86.86 and MIN-6 cells. Taken together, these results indicated that miR-6835-3p may directly modulate AdipoR 1 expression by binding to the 3'-UTR of AdipoR1 and may affect the protein expression levels of FoxO-1 and SIRT-1 in the AdipoR1 signaling pathway.

The results demonstrated that miR-6835-3p expression in high glucose-stimulated (20 mM) SU.86.86 and MIN-6 cells was reduced compared with in the control group, thus indicating that there was a change in miR-6835-3p expression in response to glucose stimulation. In addition, transfection with miR-6835-3p mimics suppressed GSIS; however, transfection with miR-6835-3p inhibitors promoted GSIS in SU.86.86 and MIN-6 cells. These findings indicated that transient transfection of cells with miR-6835-3p mimics or inhibitors altered GSIS. Furthermore, AdipoR1 overexpression regulated GSIS in SU.86.86 and MIN-6 cells, and AdipoR1 overexpression significantly enhanced the effects of miR-6835-3p inhibitors on GSIS. In addition, GSIS was reduced in response to miR-6835-3p inhibitors in the SU.86.86 and MIN-6 cell lines following AdipoR1 knockdown with a shRNA-AdipoR1. These results confirmed that AdipoR1 was a direct target of miR-6835-3p, and targeting AdipoR1 with miR-6835-3p inhibitors may promote GSIS.

In conclusion, miR-6835-3p exerted effects on insulin secretion in the SU.86.86 and MIN-6 cell lines, which were mediated by regulating the expression of AdipoR 1 and the associated signaling pathway proteins. AdipoR1 was observed to be a direct target of miR-6835-3p, thus suggesting that miR-6835-3p may be a crucial regulator of GSIS through inhibition of the AdipoR1/SIRT-1/FoxO-1 signaling pathway. The results of the present study suggested that inhibitors of miR-6835-3p may be a potential therapeutic strategy for promoting GSIS by targeting AdipoR1, and therefore may be considered an effective treatment for type 2-DM.

\section{Acknowledgements}

Not applicable.

\section{Funding}

The present study was supported by grants from the National Natural Science Foundation of China (grant nos. 30600524 and 81341067).

\section{Availability of data and materials}

The datasets used and/or analyzed during the current study are available from the corresponding author on reasonable request.

\section{Authors' contributions}

HW conducted the experiments. LJ, ZL and WW participated in the experiments. $\mathrm{CH}$ designed the experiments and wrote the paper.

\section{Ethics approval and consent to participate}

Not applicable.

\section{Patient consent for publication}

Not applicable.

\section{Competing interests}

The authors declare that they have no competing interests.

\section{References}

1. Asif M: The prevention and control the type-2 diabetes by changing lifestyle and dietary pattern. J Educ Health Promot 3: $1-8,2014$

2. Riobo Servan P: Obesity and diabetes. Nutr Hosp 28: 138-143, 2013.

3. Xiao J, Chen T and Cao H: Flavonoid glycosylation and biological benefits. Biotechnol Adv: May 22, 2014 (Epub ahead of print).

4. Bartel DP: MicroRNAs: Target recognition and regulatory functions. Cell 136: 215-233, 2009.

5. Lahmy R, Soleimani M, Sanati MH, Behmanesh M, Kouhkan F and Mobarra N: Pancreatic islet differentiation of human embryonic stem cells by microRNA overexpression. J Tissue Eng Regen Med 10: 527-534, 2016.

6. Stefani G and Slack FJ: Small non-coding RNAs in animal development. Nat Rev Mol Cell Biol 9: 219-230, 2008.

7. Shi Y and Jin Y: MicroRNA in cell differentiation and development. Sci China C Life Sci 52: 205-211, 2009.

8. Carrington JC and Ambros V: Role of microRNAs in plant and animal development. Science 301: 336-338, 2003.

9. Kaviani M, Azarpira N, Karimi MH and Al-Abdullah I: The role of microRNAs in islet $\beta$-cell development. Cell Biol Int 40: 1248-1255, 2016 
10. Dalgaard LT and Eliasson L: An 'alpha-beta' of pancreatic islet microribonucleotides. Int J Biochem Cell Biol 88: 208-219, 2017.

11. Shewade YM, Umrani M and Bhonde RR: Large-scale isolation of islets by tissue culture of adult mouse pancreas. Transplant Proc 31: 1721-1723, 1999.

12. O'Connell RM, Taganov KD, Boldin MP, Cheng G and Baltimore D: MicroRNA-155 is induced during the macrophage inflammatory response. Proc Natl Acad Sci USA 104: 1604-1609, 2007.

13. Esquela-Kerscher A and Slack FJ: Oncomirs-microRNAs with a role in cancer. Nat Rev Cancer 6: 259-269, 2006

14. Livak KJ and Schmittgen TD: Analysis of relative gene expression data using real-time quantitative PCR and the 2(-Delta Delta C(T)) method. Methods 25: 402-408, 2001

15. Nikolai VK and Steve JU: Association of RNA polymerase complexes of the parasitic protozoan cryptosporidium parvum with virus-like particles: Heterogeneous system. J Virol 74 5788-5795 2000.

16. Sebastiani G, Po A, Miele E, Ventriglia G, Ceccarelli E, Bugliani M, Marselli L, Marchetti P, Gulino A, Ferretti E and Dotta F: MicroRNA-124a is hyperexpressed in type 2 diabetic human pancreatic islets and negatively regulates insulin secretion. Acta Diabetol 52: 523-530, 2015.

17. da Silva Xavier G, Loder MK, McDonald A, Tarasov AI, Carzaniga R, Kronenberger K, Barg S and Rutter GA: TCF7L2 regulates late events in insulin secretion from pancreatic islet beta-cells. Diabetes 58: 894-905, 2009.

18. Weir GC and Bonner-Weir S: Sleeping islets and the relationship between $\beta$-cell mass and function. Diabetes 60: 2018-2019, 2011

19. Kahn SE: The relative contributions of insulin resistance and beta-cell dysfunction to the pathophysiology of type 2 diabetes. Diabetologia 46: 3-19, 2003.

20. Maris M, Ferreira GB, D'Hertog W, Cnop M, Waelkens E, Overbergh L and Mathieu C: High glucose induces dysfunction in insulin secretory cells by different pathways: A proteomic approach. J Proteome Res 9: 6274-6287, 2010.

21. Locke JM, da Silva Xavier G, Dawe HR, Rutter GA and Harries LW: Increased expression of miR-187 in human islets from individuals with type 2 diabetes is associated with reduced glucose-stimulated insulin secretion. Diabetologia 57: 122-128, 2014.

22. Ramachandran D, Roy U, Garg S, Ghosh S, Pathak S and Kolthur-Seetharam U: Sirt1 and mir-9 expression is regulated during glucose-stimulated insulin secretion in pancreatic $\beta$-islets. FEBS J 278: 1167-1174, 2011

23. Osmai M, Osmai Y, Bang-Berthelsen $\mathrm{CH}$, Pallesen EM, Vestergaard AL, Novotny GW, Pociot F and Mandrup-Poulsen T: MicroRNAS as regulators of beta-cell function and dysfunction. Diabetes Metab Res Rev 32: 334-349, 2015.
24. Thirone AC, Huang C and Klip A: Tissue-specific roles of IRS proteins in insulin signaling and glucose transport. Trends Endocrinol Metab 17: 72-78, 2006.

25. Poy MN, Eliasson L, Krutzfeldt J, Kuwajima S, Ma X, Macdonald PE, Pfeffer S, Tuschl T, Rajewsky N, Rorsman P and Stoffel M: A pancreatic islet-specific microRNA regulates insulin secretion. Nature 432: 226-230, 2004.

26. Ambros V: The functions of animal microRNAs. Nature 431: 350-355, 2004

27. Bartel DP: MicroRNAs: Genomics, biogenesis, mechanism, and function. Cell 116: 281-297, 2004.

28. Baroukh N, Ravier MA, Loder MK, Hill EV, Bounacer A, Scharfmann R, RutterGA and Van ObberghenE: MicroRNA-124a regulates Foxa2 expression and intracellular signaling in pancreatic beta-cell lines. J Biol Chem 282: 19575-19588, 2007.

29. Hu S, Wang H, Chen K, Cheng P, Gao S, Liu J, Li X and Sun X: MicroRNA-34c downregulation ameliorates-amyloid- $\beta$-induced synaptic failure and memory deficits by targeting VAMP2. J Alzheimers Dis 48: 673-686, 2015.

30. Fisman EZ and Tenenbaum A: Adiponectin: A manifold therapeutic target for metabolic syndrome, diabetes, and coronary disease? Cardiovasc Diabetol 13: 103, 2014.

31. Yamauchi T, Kamon J, Ito Y, Tsuchida A, Yokomizo T, Kita S, Sugiyama T, Miyagishi M, Hara K, Tsunoda M, et al: Cloning of adiponectin receptors that mediate antidiabetic metabolic effects. Nature 423: 762-769, 2003.

32. Yamauchi T, Iwabu M, Okada-Iwabu M and Kadowaki T: Adiponectin receptors: A review of their structure, function and how they work. Best Pract Res Clin Endocrinol Metab 28: 15-23, 2014.

33. Park HS, Lim JH, Kim MY, Kim Y, Hong YA, Choi SR, Chung S, Kim HW, Choi BS, Kim YS, et al: Resveratrol increases AdipoR1 and AdipoR2 expression in type 2 diabetic nephropathy. J Transl Med 14: 176, 2016

34. Pramanik KC, Fofaria NM, Gupta $P$ and Srivastava SK: CBP-mediated FOXO-1 acetylation inhibits pancreatic tumor growth by targeting SirtT. Mol Cancer Ther 13: 687-698, 2014.

This work is licensed under a Creative Commons Attribution-NonCommercial-NoDerivatives 4.0 International (CC BY-NC-ND 4.0) License. 